\title{
USO DE MÉTODOS ESTADÍSTICOS PARA LA IDENTIFICACIÓN DE CAPAS DE SUELOS VOLCÁNICOS CON EL ENSAYO DEL CONO DE PENETRACIÓN EN LOS TERRENOS DE LA UNIVERSIDAD DE COSTA RICA, MONTES DE OCA, SAN JOSÉ, COSTA RICA
}

\author{
USE OF STATISTICAL METHODS TO IDENTIFY LAYERS OF VOLCANIC \\ SOILS WITH THE CONE PENETRATION TEST IN A PLACE LOCATED WITHIN \\ UNIVERSIDAD DE COSTA RICA, MONTES DE OCA, SAN JOSÉ, COSTA RICA
}

\section{Rolando Mora}

Escuela Centroamericana de Geología, Universidad de Costa Rica, Apdo. 214-2060 rmorach@gmail.com

(Recibido: 15/12/2012 ; aceptado: 26/11/2013)

\begin{abstract}
Univariate (RI yT) and multivariate $\left(\mathrm{D}^{2}\right)$ statistical methods have been applied to set limits of layers on a CPTU sounding, held in soils composed of fills and volcanic deposits. The results include layered models that consider different resolutions and reduced application subjective criteria for establishing the boundaries of the layers. It is considered that the index $\mathrm{D}^{2}$ provides more accurate results.

Keywords: Geotechnics, volcanic soils, soil stratigraphy, applied statistics, applied geology.

RESUMEN: Se han aplicado métodos estadísticos univariables (RI yT) y multivariables $\left(\mathrm{D}^{2}\right)$ para establecer los límites de capas en un sondeo CPTU, realizado en suelos compuestos por rellenos y depósitos de origen volcánico. Los resultados incluyen modelos de capas de resoluciones diferentes y que consideran una aplicación reducida de criterios subjetivos para establecer los límites de las capas. Se considera que el índice $\mathrm{D}^{2}$ brinda los resultados más acertados. Palabras clave: Geotecnia, suelos volcánicos, estratigrafía de suelos, estadística aplicada, geología aplicada.
\end{abstract}

MORA, R., 2013: Uso de métodos estadísticos para la identificación de capas de suelos volcánicos con el ensayo del cono de penetración en los terrenos de la Universidad de Costa Rica, Montes de Oca, San José, Costa Rica.- Rev. Geol. Amér. Central, 49: 109-120. 


\section{INTRODUCCIÓN}

El ensayo del cono de penetración (CPT) realiza un registro de datos a intervalos muy cercanos y simultáneamente mide las señales de varios parámetros, como por ejemplo la resistencia a la penetración de la punta, la fricción lateral y la presión de poro. Al ser este instrumento una herramienta de adquisición de datos, la identificación de capas de suelos mediante métodos apropiados debe ser una prioridad. Muchas veces este tipo de identificación se realiza mediante inspección visual de los perfiles de datos y estudiando la variación de la Relación de Fricción (Rf), que relaciona la fricción lateral con la resistencia de la punta.

Los valores de $R_{f}$ en conjunto con la resistencia de la punta se usan para predecir el tipo de suelo encontrado a partir de gráficos previamente establecidos (Robertson \& Campanella, 1986) (Fig. 1). Antes de utilizar este gráfico se deben establecer las capas, y en ciertos casos una inspección visual no es suficiente para obtener una estimación bastante exacta de los límites de las mismas (Wickremesinghe \& Campanella, 1991). Estos mismos autores indican que la identificación apropiada de las capas es esencial para la toma de decisiones en lo que respecta a diseño y construcción, por lo que una estimación subjetiva puede conducir a resultados erróneos, especialmente en los casos en que las diferencias entre capas no son muy aparentes. También establecen que el gráfico de clasificación de suelos es una buena herramienta, en la medida en que las distintas capas sean determinadas con precisión y en este sentido, los métodos estadísticos brindan muy buenos resultados.

Los métodos estadísticos permiten decidir el número de capas diferentes, inspeccionando la estadística de las sub-regiones dentro de la capa principal, es decir, si el diseño requiere más detalle y sofisticación, se puede escoger un número de capas basado en límites menos críticos, mientras que para un diseño no muy exigente, el número de capas se puede basar en los picos más altos del perfil estadístico (Wickremesinghe \& Campanella, 1991).
De acuerdo con Wickremesinghe \& Campanella (1991) se debe usar una "ventana" de ancho fijo $\left(\mathrm{W}_{\mathrm{D}}\right)$, examinando la porción de datos expuesta en ella misma, donde su punto central d0 se considera como un límite potencial. La "ventana" debe moverse a lo largo del perfil de datos, en pasos iguales al intervalo de muestreo y en cada punto d0 se deben examinar las dos series de datos (una arriba y otra abajo de $\mathrm{d} 0$ ), para establecer sus diferencias con el uso de procedimientos estadísticos como el Coeficiente de Correlación Intraclase (RI) o la estadística $\mathrm{T}$ para datos de una variable, y $\mathrm{D}^{2}$ para datos de variables múltiples (Wickremesinghe \& Campanella, 1991). Según los mismos autores, la variación de las estadísticas se grafica en función de la profundidad, obteniéndose los límites de capas óptimos mediante los picos que presenten. También indican que si solo se desea establecer las capas más disímiles, se deben escoger los datos $\mathrm{d} 0$ con los valores más altos, sin embargo, si se desea una identificación de capas más elabora$\mathrm{da}$, se deben seleccionar los datos con valores moderadamente altos.

\section{Métodos estadísticos utilizados con datos de una variable}

\section{Relación T}

A cada lado del centro $\mathrm{d}_{0}$ de la ventana, habrán dos muestras, $\Omega_{1}$ y $\Omega_{2}$. Sean $\left(\bar{Q}_{1}\right)$ y $\left(\overline{Q_{2}}\right)$ las medias de las muestras y $\sigma_{1}^{2}$ y $\sigma_{2}^{2}$ las varianzas con tamaños de muestras $\mathrm{n}_{1} \mathrm{y}_{2}$ respectivamente, para cada uno de los parámetro principales del CPT.

$$
\begin{aligned}
& \sigma_{1}^{2}=\frac{1}{\left(n_{1}-1\right)} \sum_{i=1}^{n_{1}}\left(Q_{i}-\overline{Q_{1}}\right)^{2} \\
& \sigma_{2}^{2}=\frac{1}{\left(n_{2}-1\right)} \sum_{i=1}^{n_{2}}\left(Q_{i}-\overline{Q_{2}}\right)^{2}
\end{aligned}
$$


La varianza combinada intraclase $\gamma_{\mathrm{w}}{ }^{2}$ está dada por:

$\gamma_{w}^{2}=\frac{n_{1}}{n_{1}+n_{2}+1} \sigma_{1}^{2}+\frac{n_{2}}{n_{1}+n_{2}+1} \sigma_{2}^{2}$

La relación $\mathrm{T}$ fue definida por Webster \& Beckett (1968) como:

$$
T=\frac{\overline{Q_{1}}-\overline{Q_{2}}}{\gamma_{w}} \sqrt{\frac{n_{1} n_{2}}{n_{1}+n_{2}}}
$$

Para obtener una diferenciación óptima entre dos capas adyacentes, se requiere:

- que la diferencia entre las medias $\left(\overline{Q_{1}}-\bar{Q}_{2}\right)$ sea máxima,

- que las varianzas individuales de los dos segmentos $\sigma_{1}^{2}$ y $\sigma_{2}^{2}$ sean relativamente bajas, es decir, la varianza combinada intraclase será apreciablemente baja,

Según Wickremesinghe \& Campanella (1991) si se consideran los anteriores requerimientos, la relación $\mathrm{T}$ necesariamente debe presentar un pico en los límites potenciales de capas del perfil.

\section{Coeficiente de Correlación Intraclase}

Webster \& Beckett (1968) definen la varianza de la muestra combinada como:

$$
\gamma_{b}{ }^{2}=\frac{1}{n_{1}+n_{2}-1} \sum_{i=1}^{n_{1}+n_{2}}\left(Q_{i}-\bar{Q}\right)^{2}
$$

donde $\bar{Q}$ es la media de todos los datos $Q_{i}$ con $i=1,2, \ldots\left(\mathrm{n}_{1}+\mathrm{n}_{2}\right)$.

El Coeficiente de Correlación Intraclase, RI, está dado por la expresión (Wickremesinghe \& Campanella, 1991):

$$
R I=\frac{\gamma_{b}^{2}}{\gamma_{b}^{2}+\gamma_{w}^{2}}
$$

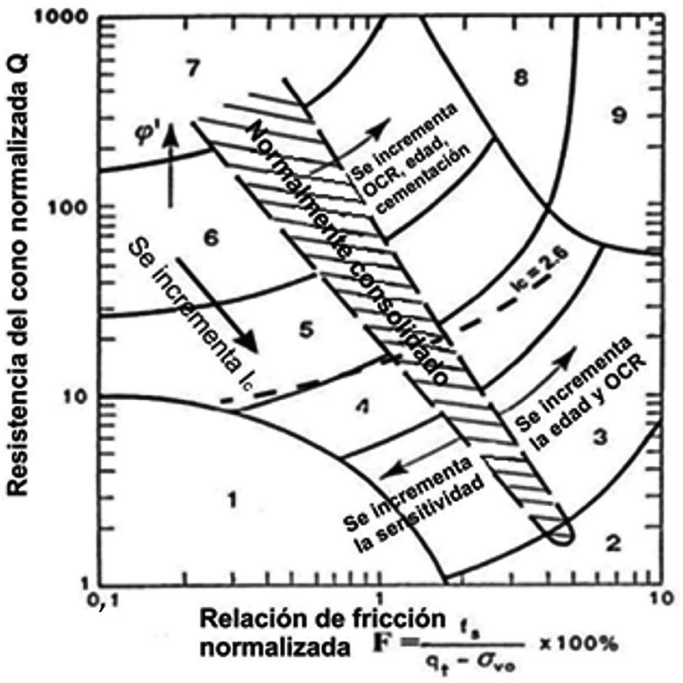

\begin{tabular}{|c|l|c|}
\hline Zona & \multicolumn{1}{|c|}{ Tipo de suelo } & $\boldsymbol{I}_{c}$ \\
\hline 1 & Sensitivo de grano fino & $\mathrm{N} / \mathrm{D}$ \\
\hline 2 & Suelos orgánicos (turbas) & $>3,6$ \\
\hline 3 & Arcillas (arcilla limosa a arcilla) & $2,95-3,6$ \\
\hline 4 & $\begin{array}{l}\text { Mezclas de limos (limo arcilloso } \\
\text { a arcilla limosa) }\end{array}$ & $2,60-2,95$ \\
\hline 5 & $\begin{array}{l}\text { Mezclas de arenas (arena limosa } \\
\text { a limo arenoso) }\end{array}$ & $2,05-2,6$ \\
\hline 6 & $\begin{array}{l}\text { Arenas (arena limpia a } \\
\text { arena limosa) }\end{array}$ & $1,31-2,05$ \\
\hline 7 & Arena gravosa a arena densa & $<1,31$ \\
\hline 8 & Arena muy rigida a arena arcillosa & $\mathrm{N} / \mathrm{D}$ \\
\hline 9 & Suelos de grano fino muy rigidos & $\mathrm{N} / \mathrm{D}$ \\
\hline
\end{tabular}

Fig. 1: Gráfico normalizado de clasificación de suelos (modificado de ASTM, 2012).

De la ecuación anterior se desprende que (Wickremesinghe \& Campanella, 1991):

- si cada muestra, $\Omega_{l}$ y $\Omega_{2}$, es absolutamente uniforme, entonces $\gamma_{\mathrm{w}}{ }^{2}$ es igual a cero y RI alcanza su máximo valor de una unidad,

- si las diferencias entre las muestras no son significativas, entonces $\gamma_{\mathrm{b}}^{2}$ y RI no son significativamente superiores a cero

- RI siempre se ubicará entre estos dos extremos y un valor relativamente alto indicará la presencia de un límite de capa en ese punto. 


\section{Método estadístico utilizado con registros multivariables}

En el ensayo CPTU se registran datos en varios canales, siendo los más importantes la resistencia del cono, la fricción lateral y la presión de poro. Cada uno de estos parámetros exhibe una clase de comportamiento distinto en los diferentes tipos de suelos, por lo tanto se requiere un método que considere todos los parámetros simultáneamente y que sea eficiente y preciso, estas exigencias las satisface una función discriminante: la estadística $\mathrm{D}^{2}$.

\section{Estadística $D^{2}$}

Se tiene una muestra $\Omega_{1}$ de m variables, la que forma un grupo de puntos en el espacio mdimensional; también se tiene otra muestra $\Omega_{2}$ que se puede describir similarmente por las mismas $\mathrm{m}$ variables en el espacio $\mathrm{m}$-dimensional. La determinación de un plano (m-1)-dimensional que separe los dos grupos de puntos es la función discriminante $\mathrm{D}^{2}$, que es la distancia entre las medias multivariables de los espacios de muestreo bidimensionales $\Omega_{1}$ y $\Omega_{2}$, lo que implica que cuanto mayor sea el valor de $\mathrm{D}^{2}$, más distintas serán las dos muestras (Rao, 1965; Harbaugh \& Merriam, 1968).

La estadística $\mathrm{D}^{2}$ está dada por la siguiente expresión (Wickremesinghe \& Campanella, 1991):

$$
D^{2}=\left\{\overline{Q_{1}}-\overline{Q_{2}}\right\}^{\prime} W^{-1}\left\{\overline{Q_{1}}-\overline{Q_{2}}\right\}
$$

donde:

$$
\{\mathrm{Q} 1-\mathrm{Q} 2\}=\left(\begin{array}{c}
\Delta \mathrm{q} \\
\Delta \mathrm{f} \\
\Delta \mathrm{u}
\end{array}\right)
$$

Es la matriz columnar ( $\mathrm{m} \times 1)$ de las diferencias entre las medias de las variables en las dos muestras. $\{\mathrm{W}\}$ es la matriz combinada de varianza-covarianza de las muestras $\Omega_{1}$ y $\Omega_{2}$.

Si se tiene una serie de $n 1$ datos a partir de $\Omega_{1}$ y $\mathrm{n}_{2}$ datos a partir de $\Omega_{2}$, estas se pueden describir con las siguientes variables: $q_{1}, f_{1}, u_{1}$ serían la resistencia, la fricción y la presión de poro en $\Omega_{1}$,

$q_{2}, f_{2}, u_{2}$ serían la resistencia, la fricción y la presión de poro en $\Omega_{2}$,

$q_{1}, f_{1}, u_{1}$, son las medias en la muestra $\Omega_{1}$, $q_{2}, f_{2}, u_{2}$ son las medias en la muestra $\Omega_{2}$, $\sigma_{\mathrm{q} 1}{ }^{2}, \sigma_{\mathrm{f} 1}{ }^{2}, \sigma_{\mathrm{u} 1}{ }^{2} \sigma_{\mathrm{q} 2}{ }^{2}, \sigma_{\mathrm{f} 2}{ }^{2}, \sigma_{\mathrm{u} 2}{ }^{2}$ son sus varianzas,

Las diferencias de las medias de las dos muestras están dadas por:

$$
\begin{aligned}
& \Delta \mathrm{q}=\mathrm{q}_{1}-\mathrm{q}_{2} \\
& \Delta \mathrm{f}=\mathrm{f}_{1}-\mathrm{f}_{2} \\
& \Delta \mathrm{u}=\mathrm{u}_{1}-\mathrm{u}_{2}
\end{aligned}
$$

Las covarianzas están dadas por:

$$
\begin{aligned}
& \sigma_{q I f 1}{ }^{2}=\frac{\sum_{i=1}^{n 1} q_{1 i} f_{1 i}}{n_{1}}-\frac{\sum_{i=1}^{n 1} q_{1 i} \sum_{i=1}^{n 1} f_{1 i}}{n_{1}{ }^{2}} \\
& \sigma_{q 1} u_{1}{ }^{2}=\frac{\sum_{i=1}^{n 1} q_{1 i} u_{1 i}}{n_{1}}-\frac{\sum_{i=1}^{n 1} q_{1 i} \sum_{i=1}^{n 1} u_{1 i}}{n_{1}{ }^{2}} \\
& \sigma f_{1} u_{1}{ }^{2}=\frac{\sum_{i=1}^{n 1} f_{1 i} u_{1 i}}{n_{1}}-\frac{\sum_{i=1}^{n 1} f_{1 i} \sum_{i=1}^{n 1} u_{1 i}}{n_{1}{ }^{2}}
\end{aligned}
$$

$$
\sigma q 2 f 2^{2}=\frac{\sum_{i=1}^{n 1} q 2_{i} f 2_{i}}{n 2}-\frac{\sum_{i=1}^{n 1} q 2_{i} \sum_{i=1}^{n 1} f 2_{i}}{n 2^{2}}
$$

$\sigma q 2 u 2^{2}=\frac{\sum_{i=1}^{n 1} q 2_{i} u 2_{i}}{n 2}-\frac{\sum_{i=1}^{n 1} q 2_{i} \sum_{i=1}^{n 1} u 2_{i}}{n 2^{2}}$

$\sigma f 2 u 2^{2}=\frac{\sum_{i=1}^{n 1} f 2_{i} u 2_{i}}{n 2}-\frac{\sum_{i=1}^{n 1} f 2_{i} \sum_{i=1}^{n 1} u 2_{i}}{n 2^{2}}$ 
Las varianzas ponderadas combinadas se expresan como:

$$
\begin{aligned}
& \Gamma_{q}^{2}=\frac{n_{1}}{n_{1}+n_{2}-1} \sigma_{q 1}^{2}+\frac{n_{2}}{n_{1}+n_{2}-1} \sigma_{q} 2^{2} \\
& \Gamma f^{2}=\frac{n_{1}}{n_{1}+n_{2}-1} \sigma f_{1}^{2}+\frac{n_{2}}{n_{1}+n_{2}-1} \sigma f 2^{2} \\
& \Gamma u^{2}=\frac{n_{1}}{n_{1}+n_{2}-1} \sigma u_{1}^{2}+\frac{n_{2}}{n_{1}+n_{2}-1} \sigma u 2^{2}
\end{aligned}
$$

De forma similar, las covarianzas ponderadas combinadas se expresan como:

$$
\begin{aligned}
& \Gamma q f^{2}=\frac{n_{1}}{n_{1}+n_{2}-1} \sigma q_{1} f 1^{2}+\frac{n_{2}}{n_{1}+n_{2}-1} \sigma q 2 f 2^{2} \\
& \Gamma q u^{2}=\frac{n_{1}}{n_{1}+n_{2}-1} \sigma q_{1} u 1^{2}+\frac{n_{2}}{n_{1}+n_{2}-1} \sigma q 2 u 2^{2} \\
& \Gamma f u^{2}=\frac{n_{1}}{n_{1}+n_{2}-1} \sigma f_{1} u 1^{2}+\frac{n_{2}}{n_{1}+n_{2}-1} \sigma f 2 u 2^{2}
\end{aligned}
$$

La matriz de varianza-covarianza fue formulada por Harbaugh \& Merriam (1968) como:

$$
\{W\}=\left(\begin{array}{ccc}
\Gamma_{q}{ }^{2} & \Gamma_{q f}{ }^{2} & \Gamma_{q u}{ }^{2} \\
\Gamma_{q f}{ }^{2} & \Gamma_{f}{ }^{2} & \Gamma_{f u}{ }^{2} \\
\Gamma_{q u}{ }^{2} & \Gamma_{f u}{ }^{2} & \Gamma_{u}{ }^{2}
\end{array}\right)
$$

De la misma manera que con los métodos de una variable, en este caso la ventana se mueve a lo largo del perfil, con el punto medio $\mathrm{d}_{0}$ separando las dos muestras y para cada d0 se calcula $\mathrm{D}^{2} \mathrm{y}$ este se grafica en función de la profundidad. Los picos del gráfico ilustran claramente las mejores posiciones de los límites de las capas; si solamente se requieren algunos límites, se seleccionan los puntos en los que se dan los valores más altos de $\mathrm{D}^{2}$, si se requieren más capas, se pueden utilizar los valores menos críticos de $\mathrm{D}^{2}$ (Wickremesinghe \& Campanella, 1991).

\section{APLICACIÓN DE LOS MÉTODOS EN UN PERFIL CPTU}

El sondeo CPTU se realizó en un sitio dentro de la propiedad de la Universidad de Costa Rica, en San Pedro de Montes de Oca (Fig. 2), en este lugar se construirán nuevas edificaciones del Alma Mater. En la figura 3 se aprecian los registros del sondeo en función de la profundidad, de esta figura se puede establecer que la diferenciación de la estratigrafía de suelos es difícil de realizar a simple vista y que se requieren técnicas que ayuden a resolver este problema.

Paralelamente a la realización de este sondeo, se ejecutó una prueba con el dilatómetro Marchetti, la cual se ha utilizado para calcular la distribución de los esfuerzos total, neutro y efectivo en función de la profundidad (Fig.4), este ensayo es de gran ayuda a la hora de establecer la estratigrafía del sitio, como se verá más adelante. La figura 4 permite apreciar que hay capas con mayor o menor presión de poro, pero esto no es criterio suficiente para definirlas.

\section{Resultados del cálculo de RI y T}

A cada una de las mediciones del ensayo CPTU sele ha aplicado el índice RI y el T, utilizando una ventana de $1.0 \mathrm{~m}$ de largo, implementada en una hoja de cálculo del programa Excel 2010.

En las figura 5, 6 y 7 se aprecian los resultados obtenidos de esta aplicación para cada uno de los parámetros medidos durante el sondeo CPTU. Si para la resistencia a la penetración del cono se consideran los picos de RI mayores o iguales a 0,8 y los picos de $\mathrm{T}$ fuera del rango de -30 a 30 (Fig. 5), se pueden establecer los límites de capa tentativos del cuadro 1 . De acuerdo con este resultado y considerando únicamente la resistencia a la penetración del cono, el RI discrimina seis capas y el T nueve, coincidiendo en un $67 \%$ de los casos.

En el caso de la fricción lateral medida durante el ensayo (Fig. 6) se han considerado los 


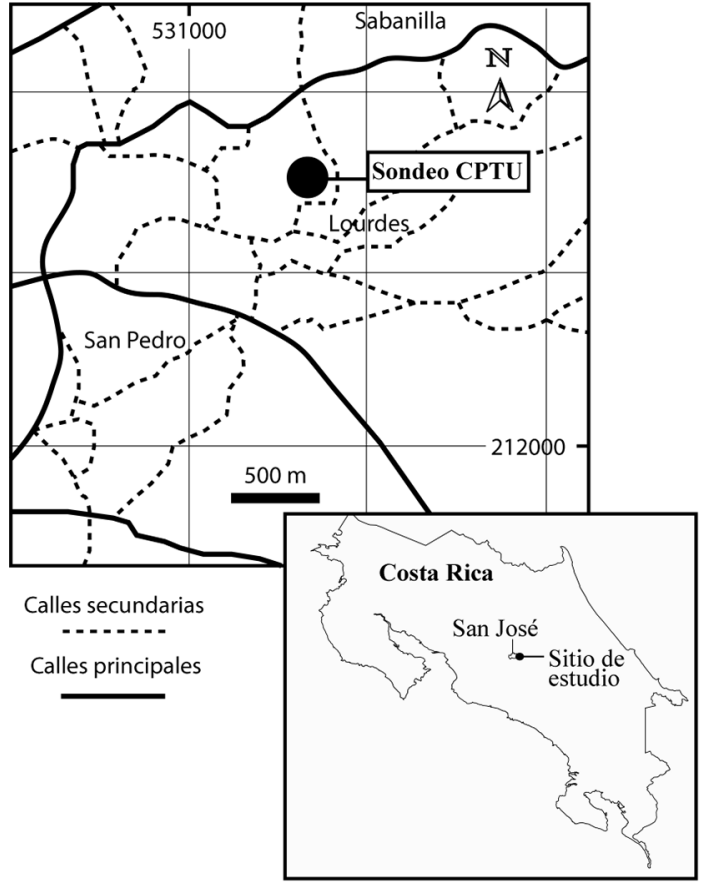

Fig. 2: Localización del sondeo CPTU en los terrenos de la Universidad de Costa Rica.

picos de RI mayores o iguales a 0,8 y los picos de $T$ fuera del rango de -400 a 400 , de manera que se establecen los límites de capas tentativos del cuadro 1 , donde se aprecia que el RI discrimina tres capas y el $\mathrm{T}$ ocho, es decir, coinciden en un $37.5 \%$ de los casos.

En el caso de la presión de poro (Fig. 7) se consideraron los picos de RI mayores o iguales a 0,8 y los picos de $\mathrm{T}$ fuera del rango de -1000 a 1000 , de manera que se establecen los límites de capas tentativos del cuadro 1 , donde se aprecia que el RI discrimina cuatro capas y el $\mathrm{T}$ cinco, es decir, coinciden en un $12.5 \%$ de los casos.

Ahora que se cuenta con un modelo de capas, se procede a calcular la relación de fricción normalizada y la resistencia normalizada del cono (Fig.1) y se grafican los valores correspondientes a cada capa, definida en el modelo anteriormente establecido (Fig. 8). Al interpretar la figura anterior se debe adoptar algún criterio, en este caso se han evaluado los porcentajes de puntos cayendo en cada ámbito del gráfico en

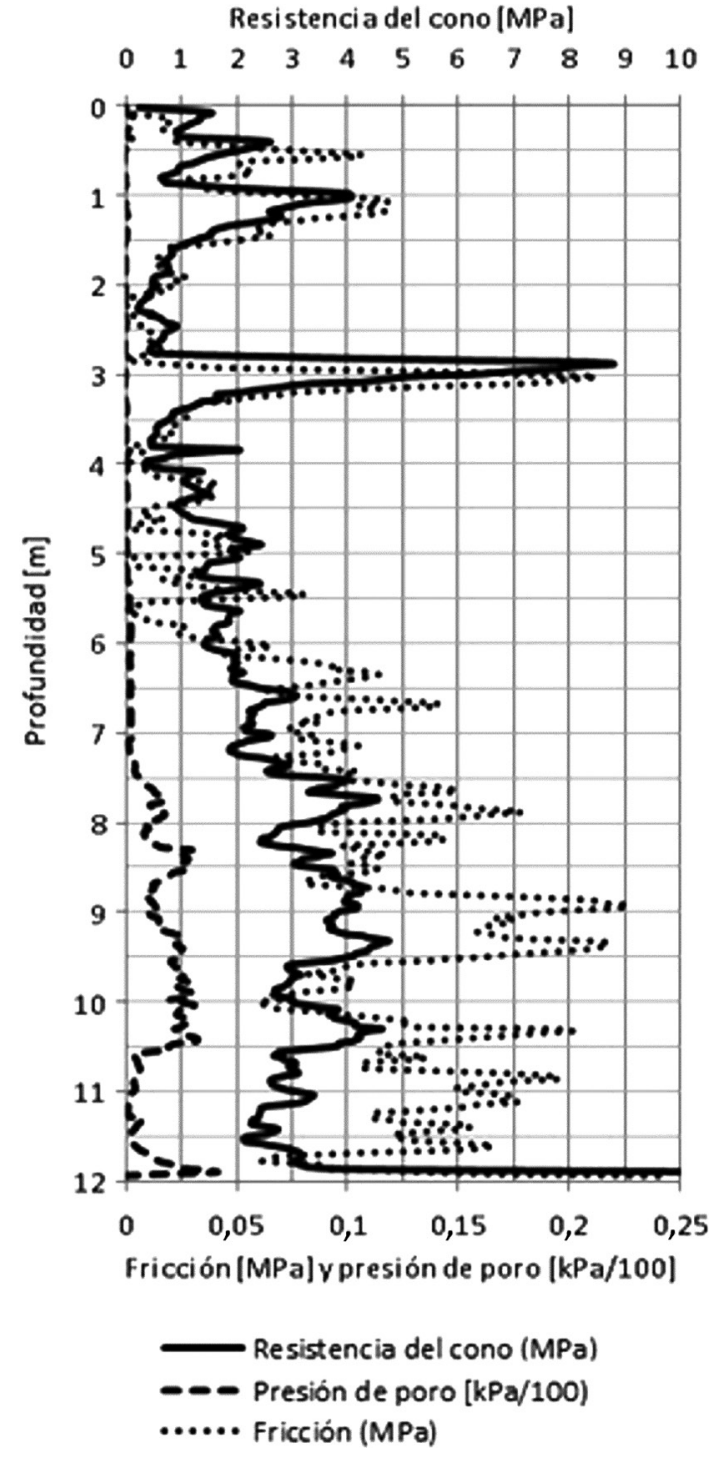

Fig. 3: Resultados del ensayo CPTU.

función de la capa analizada (Cuadro 3) y se propone la asignación de nomenclatura de acuerdo con el cuadro 4.

En general, el modelo discrimina claramente una capa de relleno, caracterizada por la dispersión de los puntos en la figura 8 , el resto de las capas se encuentran circunscritas a ámbitos bien definidos. La versión gráfica del modelo final se presenta en la figura 9, acompañada de la distri- 
Cuadro 1

Límites de capa tentativos para la resistencia a la penetración del cono, fricción lateral y presión de poro, de acuerdo con los índices RI y T

\begin{tabular}{cccccc}
\hline \multicolumn{2}{c}{ Resistencia a la penetración del cono } & \multicolumn{2}{c}{ Fricción lateral } & \multicolumn{2}{c}{ Presión de poro } \\
\hline RI & T & RI & T & RI & T \\
\hline Profundidad [m] & Profundidad [m] & Profundidad $[\mathrm{m}]$ & Profundidad [m] & Profundidad [m] & Profundidad [m] \\
\hline ND & 1,88 & 1,48 & 1,48 & ND & 2,62 \\
4,62 & 4,62 & ND & 2,06 & ND & 3,26 \\
ND & 6,44 & ND & 3,7 & ND & 3,98 \\
7,46 & 7,46 & ND & 4,12 & 5,3 & 5,3 \\
8,5 & 8,5 & ND & 6,14 & ND & 6,14 \\
9,54 & 9,54 & ND & 7,48 & 7,5 & ND \\
10,04 & 10,04 & 8,78 & 8,78 & 9,2 & ND \\
10,52 & 10,52 & 9,54 & 9,54 & 10,54 & ND \\
ND & 11,12 & - & - & - & -
\end{tabular}

ND: capa no detectada

bución en profundidad de los esfuerzos, para así poder apreciar donde se presentan los límites de las capas.

\section{Resultados del cálculo del $\mathrm{D}^{2}$}

En cálculo del índice D2 se ha utilizado una ventana también de $1.0 \mathrm{~m}$ de largo y se ha utilizado la hoja de calculo del programa Excel 2010, los resultados obtenidos se observan en la figura 10. Para seleccionar los picos, es decir, los límites de capa, se ha establecido considerar los que se encuentren fuera del rango entre -40 y 40, con lo cual se obtiene el modelo de siete capas del cuadro 5. Es importante señalar que el índice D2 detecta de manera inequívoca la presencia del nivel freático a la profundidad de $5,3 \mathrm{~m}$.

Con base en las capas definidas por este modelo, se grafican las mediciones normalizadas de la fricción y la resistencia del cono por capa (Fig. 11), se evalúan los porcentajes de puntos en cada ámbito definido en el gráfico (Cuadro 6) y se propone la asignación de nomenclatura de acuerdo con el cuadro 4.
Este modelo identifica un rellano de mayor espesor, el cual se puede dividir en cuatro partes bien diferenciadas por su ubicación en el gráfico de la figura 11, luego del relleno se establecen tres capas claramente delimitadas por su ubicación en los ámbitos de la figura 11. Finalmente, en la figura 12 se presenta gráficamente el segundo modelo, junto con la distribución de los esfuerzos en profundidad.

Si comparamos las figuras 9 y 12 se nota que el índice D2 logra discriminar un relleno de mayor espesor y cuatro capas dentro de este, incluyendo la capa de arcilla limosa establecida por el modelo basado en RI y T. Además, las capas de limo arenoso con algo de arcilla y limo arenoso del modelo basado en RI y T, es interpretada como una sola capa de limo arenoso con cantidad menor de arcilla por el modelo D2. Finalmente, en la parte más profunda del sondeo el modelo D2 discrimina dos capas donde el otro modelo solo identifica una capa.

Del uso de los dos modelos, se puede decir que el modelo basado en RI yT no elimina totalmente la subjetividad a la hora de establecer los límites de las capas, desde esta perspectiva, el modelo $\mathrm{D}^{2}$ ofrece una alternativa menos subjeti- 


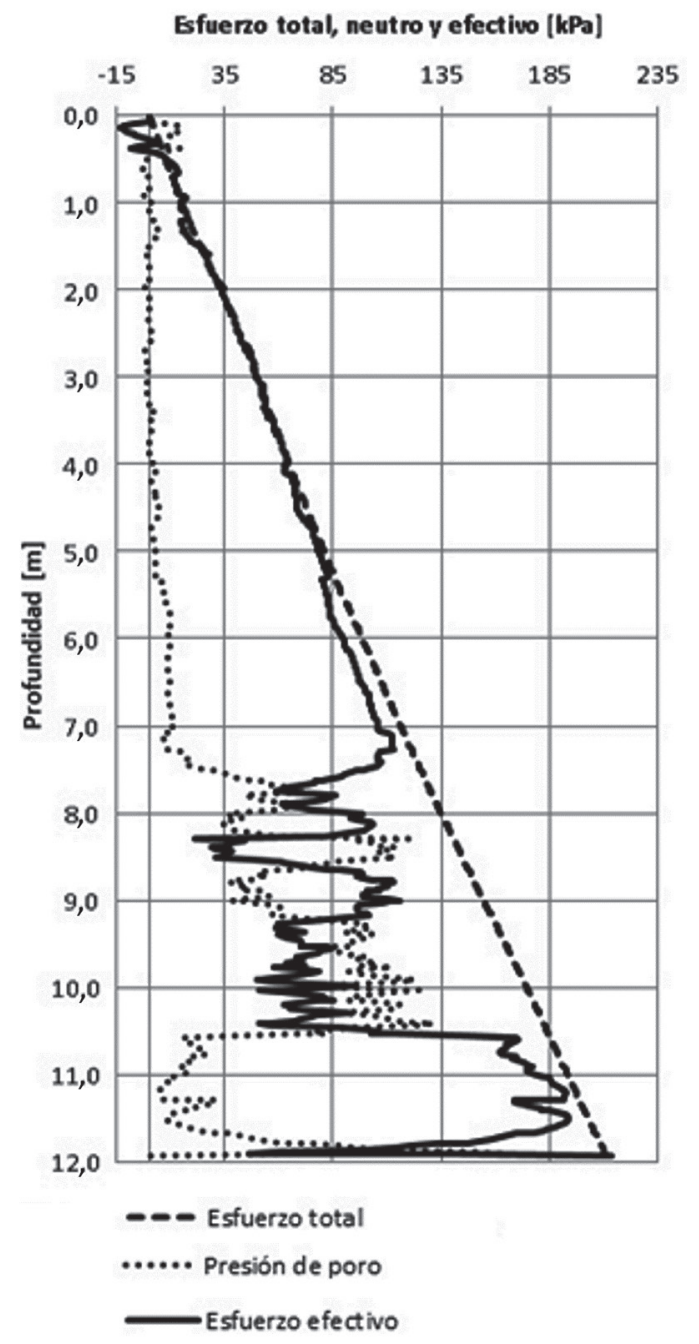

Fig. 4: Distribución de los esfuerzos total, neutro y efectivo en función de la profundidad.

\section{Cuadro 2}

Modelo de cinco capas con base en la aplicación de los índices RI yT a los parámetros medidos durante el sondeo CPTU

\begin{tabular}{lll}
\hline \# de capa & Limite $[\mathrm{m}]$ & Espesor $[\mathrm{m}]$ \\
\hline 1 & 6,14 & 6,14 \\
2 & 7,46 & 1,32 \\
3 & 9,54 & 2,08 \\
4 & 10,52 & 0,98 \\
5 & 11,34 & 0,82 \\
\hline
\end{tabular}

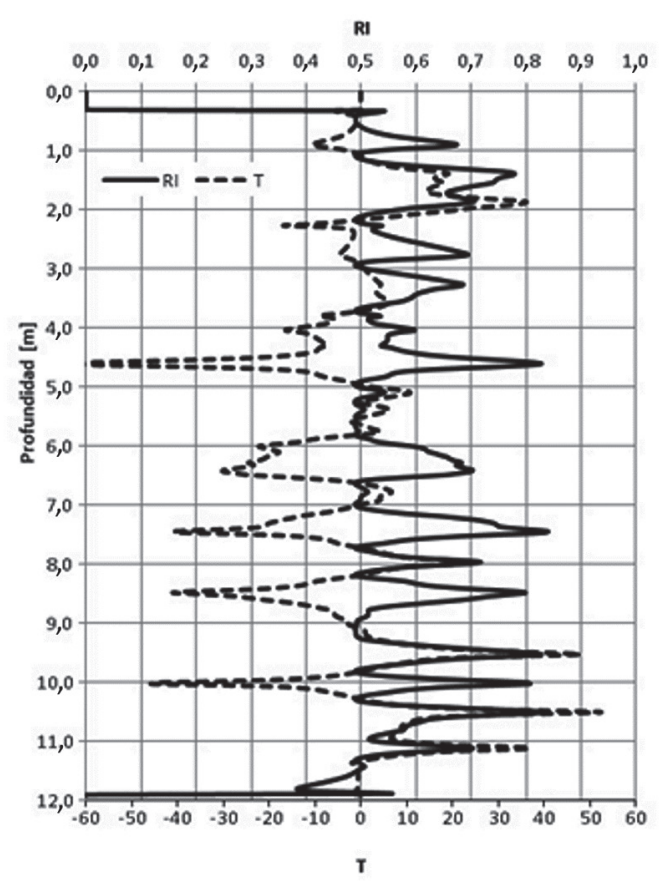

Fig. 5: Resultados de RI y T para la resistencia a la penetración del ensayo CPTU.

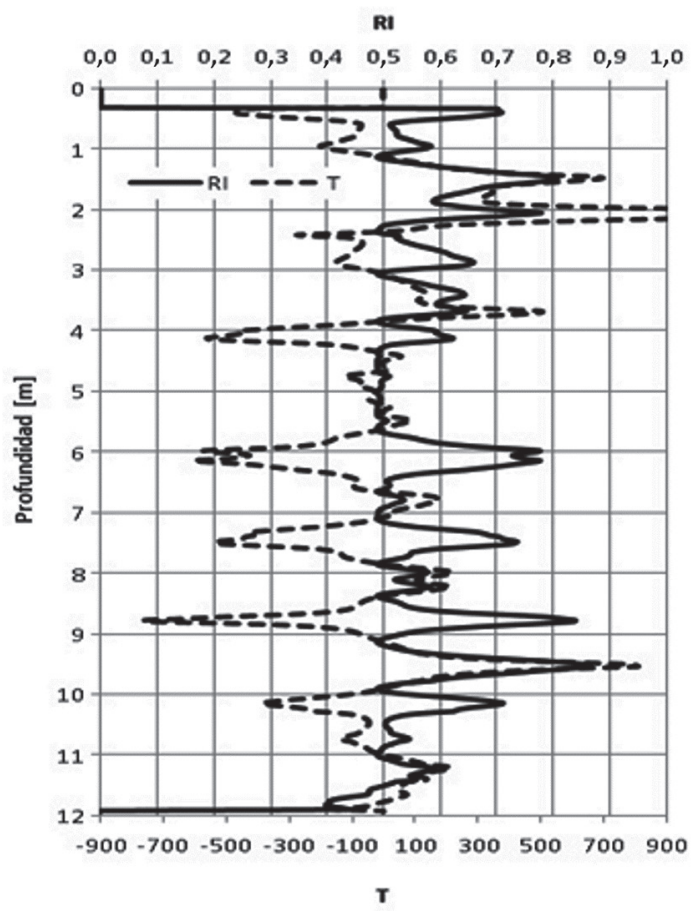

Fig.6: Resultados de RI y T para la fricción lateral del ensayo CPTU. 


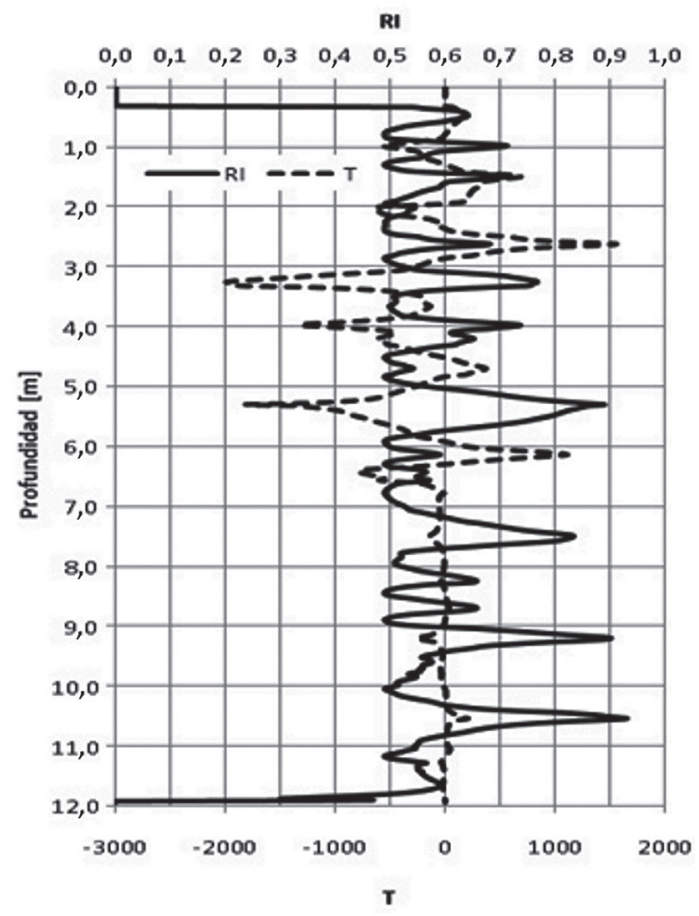

Fig.7: Resultados de RI y T para la presión de poro del ensayo CPTU.

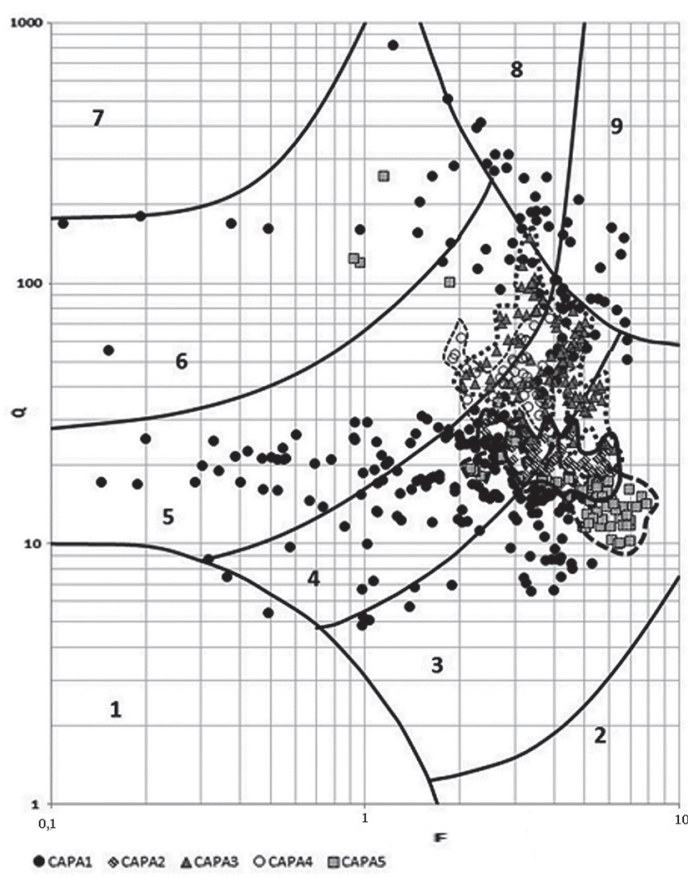

Fig.8: Discriminación por ámbitos de los puntos pertenecientes a cada capa del primer modelo.

Cuadro 3

Evaluación de las capas de acuerdo con el número de puntos presentes en cada ámbito del gráfico de la figura 8

\begin{tabular}{|c|c|c|c|c|c|c|c|c|c|c|}
\hline \multirow[b]{2}{*}{ Suelo } & \multicolumn{2}{|c|}{ Capa 1} & \multicolumn{2}{|c|}{ Capa 2} & \multicolumn{2}{|c|}{ Capa 3} & \multicolumn{2}{|c|}{ Capa 4} & \multicolumn{2}{|c|}{ Capa 5} \\
\hline & $\begin{array}{c}\# \text { de } \\
\text { puntos }\end{array}$ & {$[\%]$} & $\begin{array}{l}\text { \# de } \\
\text { puntos }\end{array}$ & {$[\%]$} & $\begin{array}{c}\text { \# de } \\
\text { puntos }\end{array}$ & {$[\%]$} & $\begin{array}{c}\# \text { de } \\
\text { puntos }\end{array}$ & {$[\%]$} & $\begin{array}{l}\text { \# de } \\
\text { puntos }\end{array}$ & {$[\%]$} \\
\hline $\begin{array}{l}\text { Sensitivo de } \\
\text { grano fino }\end{array}$ & 2 & 0,7 & 0 & 0,0 & 0 & 0,0 & 0 & 0,0 & 0 & 0,0 \\
\hline Orgánico & 0 & 0,0 & 0 & 0,0 & 0 & 0,0 & 0 & 0,0 & 0 & 0,0 \\
\hline Arcilla & 59 & 21,9 & 42 & 63,6 & 17 & 16,3 & 0 & 0,0 & 57 & 83,8 \\
\hline Limo & 116 & 43,1 & 24 & 36,4 & 59 & 56,7 & 34 & 69,4 & 10 & 14,7 \\
\hline $\begin{array}{l}\text { Arenas con } \\
\text { finos }\end{array}$ & 49 & 18,2 & 0 & 0,0 & 28 & 26,9 & 15 & 30,6 & 1 & 1,5 \\
\hline Arenas limpias & 12 & 4,5 & 0 & 0,0 & 0 & 0,0 & 0 & 0,0 & 0 & 0,0 \\
\hline Arena gravosa & 0 & 0,0 & 0 & 0,0 & 0 & 0,0 & 0 & 0,0 & 0 & 0,0 \\
\hline $\begin{array}{l}\text { Suelos gruesos } \\
\text { rígidos }\end{array}$ & 18 & 6,7 & 0 & 0,0 & 0 & 0,0 & 0 & 0,0 & 0 & 0,0 \\
\hline $\begin{array}{l}\text { Suelos finos } \\
\text { rígidos }\end{array}$ & 13 & 4,8 & 0 & 0,0 & 0 & 0,0 & 0 & 0,0 & 0 & 0,0 \\
\hline Total & 269 & $100 \%$ & 66 & $100 \%$ & 104 & $100 \%$ & 49 & $100 \%$ & 68 & $100 \%$ \\
\hline Clasificación: & & & Arcill & mosa & $\begin{array}{l}\text { Limo a } \\
\text { algo } c\end{array}$ & $\begin{array}{l}\text { oso con } \\
\text { rcilla }\end{array}$ & Limo & enoso & $\begin{array}{r}\text { Arcilla } \\
\text { limo y tre }\end{array}$ & $\begin{array}{l}\text { algo de } \\
\text { de arena }\end{array}$ \\
\hline
\end{tabular}




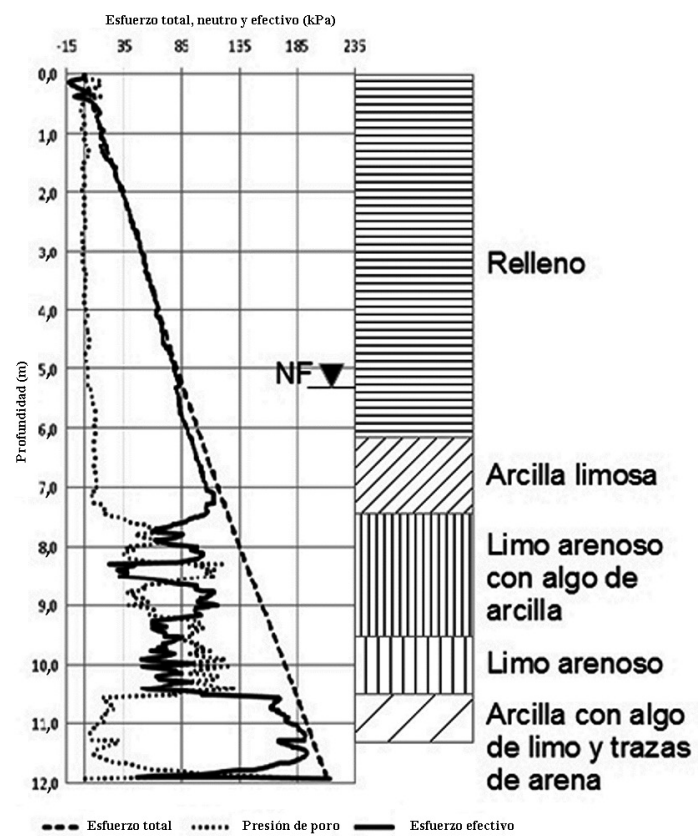

Fig. 9: Modelo de capas desarrollado mediante la utilización de los índices RI y T.

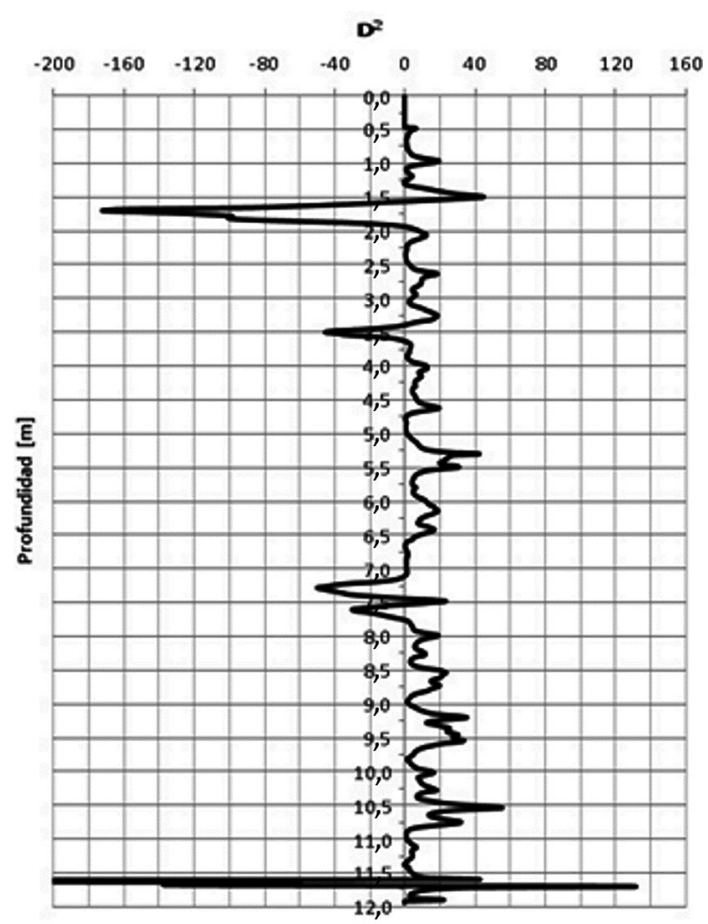

Fig. 10: resultados del cálculo del índice $\mathrm{D}^{2}$.

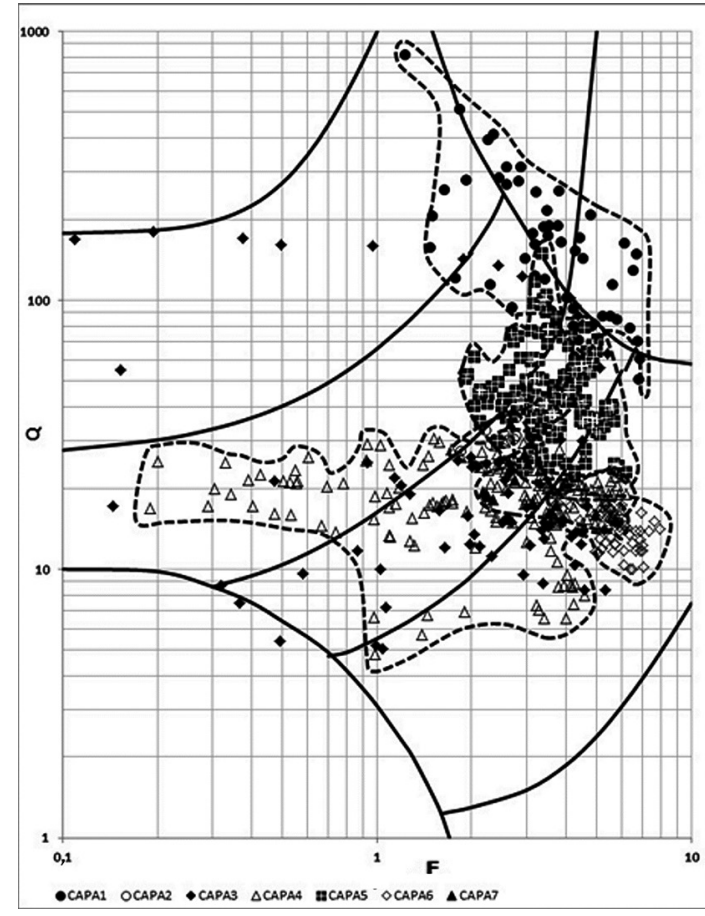

Fig.11: Discriminación por ámbitos de los puntos pertenecientes a cada capa del segundo modelo.

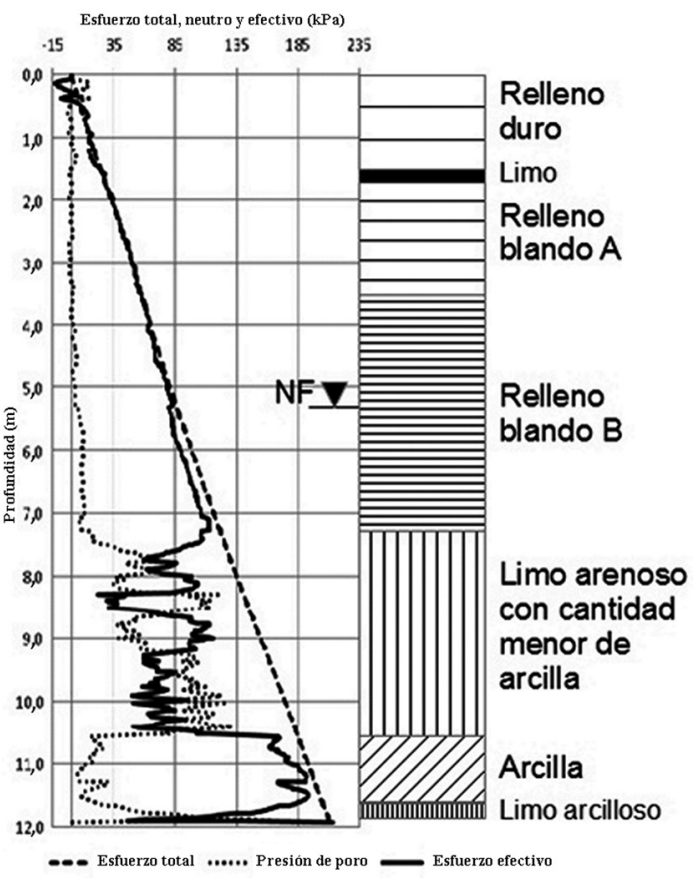

Fig. 12: Modelo de capas desarrollado mediante la utilización del índice $\mathrm{D}^{2}$. 
Cuadro 6

Evaluación de las capas de acuerdo con el número de puntos presentes en cada ámbito del gráfico de la figura 11

\begin{tabular}{|c|c|c|c|c|c|c|c|c|c|c|c|c|c|c|}
\hline \multirow[b]{2}{*}{ Suelo } & \multicolumn{2}{|c|}{ Capa 1} & \multicolumn{2}{|c|}{ Capa 2: Limo } & \multicolumn{2}{|c|}{ Capa 3} & \multicolumn{2}{|c|}{ Capa 4} & \multicolumn{2}{|c|}{ Capa 5} & \multicolumn{2}{|c|}{ Capa 6} & \multicolumn{2}{|c|}{ Capa 7} \\
\hline & $\begin{array}{c}\# \text { de } \\
\text { puntos }\end{array}$ & {$[\%]$} & $\begin{array}{c}\# \text { de } \\
\text { puntos }\end{array}$ & {$[\%]$} & $\begin{array}{c}\# \text { de } \\
\text { puntos }\end{array}$ & {$[\%]$} & $\begin{array}{c}\# \text { de } \\
\text { puntos }\end{array}$ & {$[\%]$} & $\begin{array}{c}\# \text { de } \\
\text { puntos }\end{array}$ & {$[\%]$} & $\begin{array}{c}\# \text { de } \\
\text { puntos }\end{array}$ & {$[\%]$} & $\begin{array}{c}\# \text { de } \\
\text { puntos }\end{array}$ & {$[\%]$} \\
\hline $\begin{array}{c}\text { Sensitivo } \\
\text { de grano } \\
\text { fino }\end{array}$ & 0 & 0,0 & 0 & 0,0 & 2 & 2,4 & 0 & 0,0 & 0 & 0,0 & 0 & 0,0 & 0 & 0,0 \\
\hline Orgánico & 0 & 0,0 & 0 & 0,0 & 0 & 0,0 & 0 & 0,0 & 0 & 0,0 & 0 & 0,0 & 0 & 0,0 \\
\hline Arcilla & 2 & 3,4 & 0 & 0,0 & 22 & 26,5 & 75 & 42,9 & 19 & 11,7 & 54 & 100,0 & 3 & 25,0 \\
\hline Limo & 11 & 19,0 & 10 & 100,0 & 45 & 54,2 & 67 & 38,3 & 101 & 62,0 & 0 & 0,0 & 9 & 75,0 \\
\hline $\begin{array}{l}\text { Arenas } \\
\text { con finos }\end{array}$ & 9 & 15,5 & 0 & 0,0 & 7 & 8,4 & 33 & 18,9 & 43 & 26,4 & 0 & 0,0 & 0 & 0,0 \\
\hline $\begin{array}{l}\text { Arenas } \\
\text { limpias }\end{array}$ & 5 & 8,6 & 0 & 0,0 & 7 & 8,4 & 0 & 0,0 & 0 & 0,0 & 0 & 0,0 & 0 & 0,0 \\
\hline $\begin{array}{c}\text { Arena } \\
\text { gravosa }\end{array}$ & 0 & 0,0 & 0 & 0,0 & 0 & 0,0 & 0 & 0,0 & 0 & 0,0 & 0 & 0,0 & 0 & 0,0 \\
\hline $\begin{array}{l}\text { Suelos } \\
\text { gruesos } \\
\text { rígidos }\end{array}$ & 18 & 31,0 & 0 & 0,0 & 0 & 0,0 & 0 & 0,0 & 0 & 0,0 & 0 & 0,0 & 0 & 0,0 \\
\hline $\begin{array}{l}\text { Suelos } \\
\text { finos } \\
\text { rígidos }\end{array}$ & 13 & 22,4 & 0 & 0,0 & 0 & 0,0 & 0 & 0,0 & 0 & 0,0 & 0 & 0,0 & 0 & 0,0 \\
\hline Total & 58 & $100 \%$ & 10 & $100 \%$ & 83 & $100 \%$ & 175 & $100 \%$ & 163 & $100 \%$ & 54 & $100 \%$ & 12 & $100 \%$ \\
\hline $\begin{array}{l}\text { Clasifi- } \\
\text { cación }\end{array}$ & Rellen & duro & Li1 & & Relleno & lando & Relleno & lando & $\begin{array}{l}\text { Limo a } \\
\text { con ca } \\
\text { menor d }\end{array}$ & $\begin{array}{l}\text { noso } \\
\text { idad } \\
\text { arcilla }\end{array}$ & Arc & & Limo a & illoso \\
\hline
\end{tabular}

Cuadro 4

Propuesta para la asignación de nomenclatura de las capas

\begin{tabular}{cl}
\hline$\%$ de presencia en el ámbito & \multicolumn{1}{c}{ Nomenclatura } \\
\hline$>=40 \%$ & Constituyente principal \\
$20-40 \%$ & Agregar sufijo: -oso,- osa \\
$12-20 \%$ & Agregar: con algo de... \\
$5-12 \%$ & Agregar: con cantidad \\
$<5 \%$ & menor de... \\
& Agregar: con trazas de... \\
\hline
\end{tabular}

\section{Cuadro 5}

Modelo de siete capas con base en la aplicación del índice $\mathrm{D}^{2}$ a los parámetros medidos durante el sondeo CPTU

\begin{tabular}{ccc}
\hline \# de capa & Limite $[\mathrm{m}]$ & Espesor $[\mathrm{m}]$ \\
\hline 1 & 1,50 & 1,50 \\
2 & 1,70 & 0,20 \\
3 & 3,50 & 1,80 \\
4 & 7,28 & 3,78 \\
5 & 10,54 & 3,26 \\
6 & 11,62 & 1,08 \\
7 & 11,86 & 0,24 \\
\hline
\end{tabular}


va para establecer los límites de las capas y una mayor resolución en el número de capas, pues se trata de un método multivariable.

\section{CONCLUSIONES}

Analizando los registros del sondeo CPTU en función de la profundidad, es claro que la diferenciación de la estratigrafía de suelos es difícil de realizar a simple vista y que se requieren técnicas que ayuden a resolver este problema.

La distribución de los esfuerzos total, neutro y efectivo en profundidad no es un criterio suficiente para establecer la estratigrafía del sitio de estudio, pero sirve como complemento a la hora de aplicar métodos estadísticos para lograr ese propósito.

Si se aplican los índices RI y $\mathrm{T}$ a los registros individuales de los parámetros medidos en el ensayo CPTU del sitio de estudio, se puede establecer un modelo de cinco capas, a partir de las coincidencias en la detección de límites por ambos índices, lo anterior requiere algo de subjetivismo a la hora de considerar las coincidencias.

La aplicación del índice D2 conduce a delimitar siete capas, a mejorar la resolución de capas detectadas, principalmente en el sector compuesto por relleno y a requerir mucho menor subjetivismo a la hora de establecer los límites.

Se ha propuesto una metodología sencilla, basada en la el número de mediciones que se ubican en cada ámbito del gráfico de fricción normalizada contra resistencia del cono normalizada, la cual permite asignar de manera ágil la nomenclatura de las capas detectadas.

Es importante, a la hora de aplicar los índices y delimitar las capas, analizar el registro de presión de poro para así establecer la profun- didad a la que se encuentra el nivel freático, de manera que el mismo no se confunda con un límite de capas.

\section{REFERENCIAS BIBLIOGRÁFICAS}

ASTM, 2012: ASTM D6067-10 Standard Practice for Using the Electronic Piezocone Penetrometer Tests for Environmental Site Characterization.- http://enterprise.astm.org/ filtrexx40.cgi?REDLINE_PAGES/D6067. htm [consulta: 01 de setiembre de 2012].

HARBAUGH, J.W. \& MERRIAM, D.F., 1968: Computer Applications in Stratigraphic Analysis.- 282 págs. John Wiley and Sons, New York.

RAO, C.R., 1965: Linear Statistical Inference and Its Applications.- 105 págs. John Wiley and Sons, New York.

ROBERTSON, P.K. \& CAMPANELLA, R.G., 1986: Guidelines for use, interpretation and application of CPT and CPTU.- 23 págs. Soil Mechanics Series, No. 105, Deparment of Civil Engineering, University of British Columbia, Vancouver.

WEBSTER, R. \& BECKETT, P.H.T., 1968: Quality and Usefulness of Soil Maps.Nature, 219: 680-682.

WICKREMESINGHE, D. \& CAMPANELLA, R.G., 1991: Statistical methods for soil layer boundary location using the cone penetration test.- Proc. ICASP6, Mexico City, 2, 636-643 